\title{
Il centenario del pH (1909-2009). \\ Ma in medicina, è proprio indispensabile utilizzare i logaritmi negativi per misurare gli idrogenioni? Parte I
}

The $100^{\text {th }}$ anniversary of $\mathrm{pH}$ (1909-2009). Negative logarithms for measuring hydrogen ions: are they essential in medicine? Part I

\section{Francesco Sgambato*, Sergio Prozzo, Ester Sgambato, Rosa Sgambato, Luca Milano}

UO Medicina Interna, Ospedale Fatebenefratelli, Benevento

Ricevuto il 21 dicembre 2010; accettato il 17 febbraio 2011 disponibile online il 14 aprile 2011

\section{KEYWORDS \\ $\mathrm{pH}$; \\ History of $\mathrm{pH}$; \\ Centenary of $\mathrm{pH}$; \\ Acid-base balance; \\ Equation of Henderson; \\ Equation of Henderson- \\ Hasselbalch; \\ Logarithms in Medicine.}

\begin{abstract}
Introduction: It has been 100 years since the concept of pH (1909-2009) was "invented" by the Danish chemist-mathematician Søren Peter Lauritz Sørensen (1868-1939) in the chemistry laboratories of the Carlsberg Brewery in Copenhagen. The anniversary provides an opportunity to examine the crucial importance in human life of acid-base balance.

Materials and methods: The authors review the historical process that led to the creation of the $\mathrm{pH}$ scale, with citation of passages from the original work of Sørensen published 100 years ago. This is followed by a critical analysis of the debate regarding the use of logarithms to measure hydrogen ion concentrations based on data from scientific papers published over the past 50 years (1960-2010). Results and discussion: The authors conclude that the concept of acid-base balance can be approached and taught in a simpler, more exciting, and even pleasant fashion without using the infamous and abstruse Henderson-Hasselbalch equation. The whole rationale underlying the understanding and clinical application of this vital topic is clearly and unquestionably inherent simpler, more manageable formula introduced by Henderson (without logs), which is useful and quite adequate for use in medical education.

(c) 2011 Elsevier Srl. All rights reserved.
\end{abstract}

* Corrispondenza: Ospedale Fatebenefratelli, viale Principe di Napoli 14/A - 82100 Benevento.

E-mail: sgambatof@gmail.com (F. Sgambato). 


\section{Introduzione}

Correva l'anno 1909, appena un secolo fa, quando il chimico danese Søren Peter Lauritz Sørensen (1868-1939) ( fig. 1) creò la scala numerica utile per misurare la carica degli ioni idrogeno presenti in una soluzione (ovvero per stabilire il suo grado di acidità o di alcalinità); con questa brillante idea divenne l'inventore del $\mathrm{pH}$.

Sørensen ottenne il prestigioso e storico risultato lavorando, in qualità di direttore dal 1901 al 1938, presso il Dipartimento di Chimica del laboratorio dell'azienda Carlsberg di Copenhagen. Ebbene sì, trattasi proprio della ditta produttrice della birra Carlsberg (Carlsberg Brewery) in Danimarca, sorta nel 1876, il cui laboratorio tanto ha contribuito alla ricerca sperimentale della chimica mondiale, non solo nell'ambito del rapporto acido-base [1,2].

Sørensen, in particolare, stava seguendo, in chiave pionieristica, cinque filoni di ricerca:

- la sintesi degli aminoacidi;

- gli studi analitici generali;

- la determinazione della concentrazione degli ioni idrogeno:

- gli studi sulle proteine;

- l'azione degli acidi sulle reazioni enzimatiche.

I risultati delle sue ricerche sugli ioni idrogeno furono riportati in un lavoro del 1909, divenuto una classica pietra

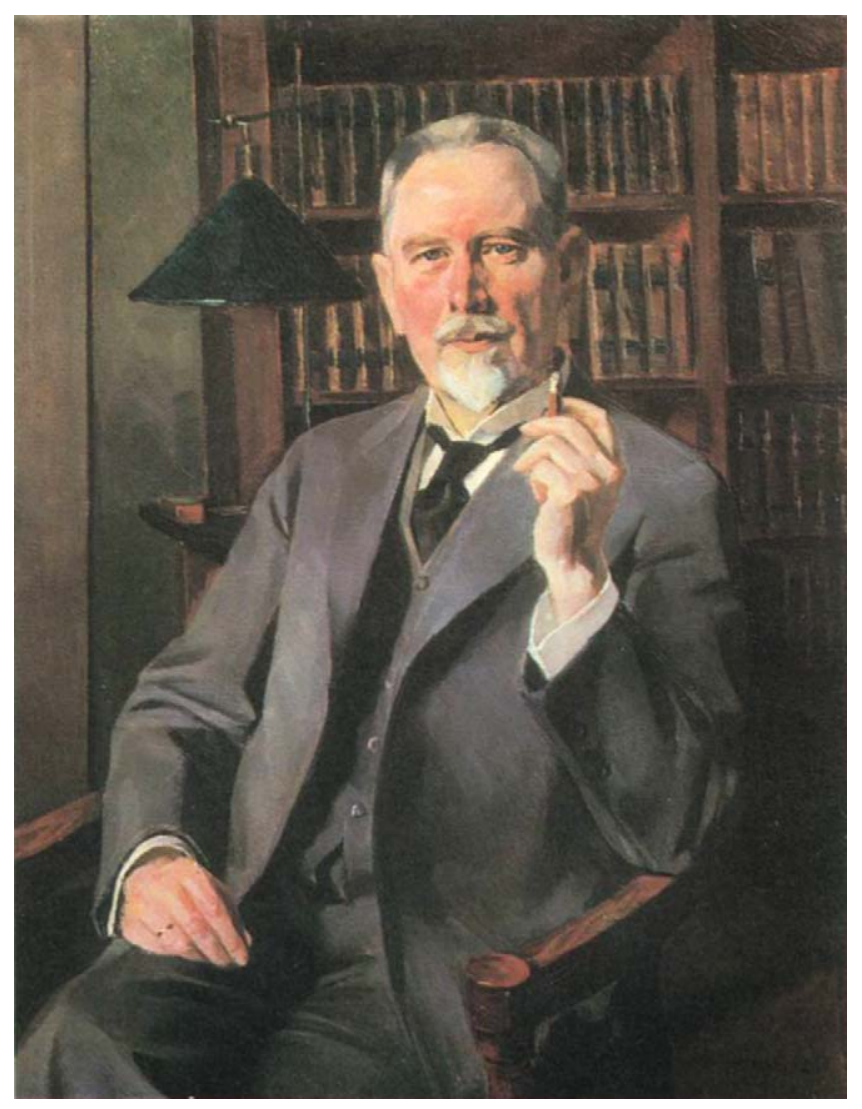

Figura 1 Søren Peter Lauritz Sørensen (1868-1939) in un ritratto all'età di 59 anni (per gentile concessione di Carlsberg Library) (fonte: Holter $\mathrm{H}$, et al. Carlsberg Foundation. Copenhagen: Rhodos, 1976).

\section{ÉTUDES ENZYMATIQUES.}

II. Sur la mesure et l'importance de la concentration des ions hydrogène dans les réactions enzymatiques.

PAR

S. P. L. SøRENSEN

Compt Rend Trav Carlsberg Lab 1909, 8, 1-168

Figura 2 Frontespizio del lavoro di Sørensen nell'edizione francese (fonte: Sørensen SPL. Compt Rend Trav Carlsberg Lab 1909;8:1-168).

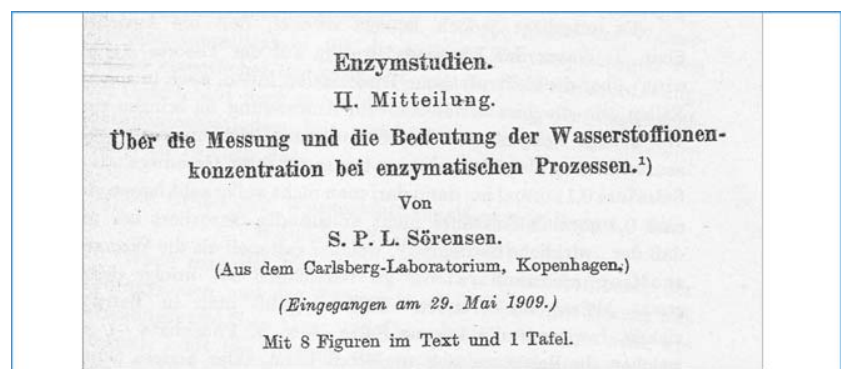

Biochem. Zeitschr. 1909, 21, 131-304 and 22, 352-356

Figura 3 Frontespizio del lavoro di Sørensen nell'edizione tedesca (fonte: Sørensen SPL. Biochem Zeitschr 1909;21: 131-304).

miliare della ricerca mondiale, dal titolo: Studi enzimatici. II. Sulla misura e l'importanza della concentrazione degli ioni idrogeno nelle reazioni enzimatiche, pubblicato contemporaneamente in tre lingue: francese, tedesco e danese [3-6] ( figg. 2,3).

Nell'introduzione al suo lavoro, Sørensen fissò alcuni concetti chiave, già nelle primissime righe.

"Si sa che la velocità, con la quale avviene una scissione enzimatica, è funzione, tra l'altro, del grado di acidità o di alcalinità dell'ambiente [milieu] in cui essa avviene."

"La concentrazione degli idrogenioni è un fattore essenziale in tutti i processi biologici."

Nella maggior parte delle condizioni studiate in biologia, la concentrazione degli ioni idrogeno in una soluzione acquosa è molto bassa; infatti, il numero di ioni idrogeno, espresso in grammi-atomi per litro (moli per litro), è inferiore a 1 e viene indicato, pertanto, con uno zero seguito da una virgola $(0$,$) e con molti altri zeri dopo la virgola ( fig. 4).$

Questi numeri decimali "ingombranti" possono anche essere scritti in un altro modo, mediante l'utilizzo dell" esponente negativo" (numero scritto tipograficamente con un carattere più piccolo in alto a destra del numero 10), il che esprime il numero di volte che la virgola deve essere spostata verso sinistra per ottenere il numero decimale desiderato ( fig. 5). Com'è facilmente intuibile, questi numeri sono di difficile gestione in tutti i calcoli matematici; tanto più lo erano in un'epoca storica in cui non erano ancora disponibili i calcolatori. Sembrò, quindi, molto ragionevole trasformare in chiave logaritmica tali numeri "scomodi", ottenendo così numeri più maneggevoli nella pratica corrente. 


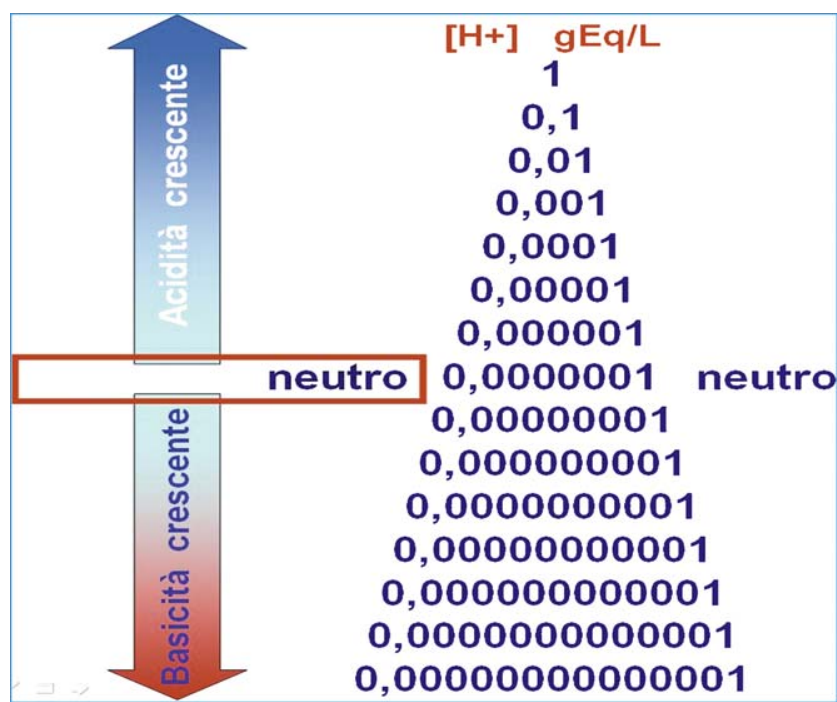

Figura 4 Le concentrazioni degli ioni idrogeno nelle soluzioni acquose sono sempre molto basse.

Nel 1909 fu proprio Sørensen, nell'esprimere numericamente la concentrazione idrogenionica delle soluzioni acquose in studio nel suo laboratorio, a suggerire la rimozione dello "scomodo esponente negativo" e a creare, così, la più conveniente scala del $\mathrm{pH}$ in chiave logaritmica da 0 a 14 (fig. 6). In questa scala, ogni passaggio da un valore di concentrazione idrogenionica al valore successivo (o al precedente) esprime una variazione pari a 10 volte in termini di quantità, mentre il pH varia di 1 sola unità, ma in senso inverso. Infatti, tra il pH e la concentrazione idrogenionica $\left[\mathrm{H}^{+}\right]$esiste un rapporto inverso, ovvero maggiore è l'aumento di $\mathrm{H}^{+}$, maggiore è la diminuzione del $\mathrm{pH}$ e viceversa, secondo un andamento logaritmico.

Ciò significa che la variazione di un'unità nella scala del pH corrisponde a una variazione dell'acidità pari a 10 volte, una variazione di 2 unità del $\mathrm{pH}$ corrisponde a una variazione pari a 100 volte, mentre una variazione di 3 unità corrisponde a una modifica dell'acidità pari a 1.000 volte, e così via in progressione. Per esempio, una soluzione idroelettrolitica avente $\mathrm{pH}$ pari a 7 ha una concentrazione idrogenionica 10

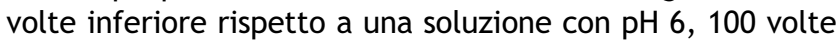
inferiore rispetto a una soluzione con pH 5 e 1.000 volte inferiore rispetto a una soluzione con $\mathrm{pH}$ di 4 ecc.

\section{Metodo di misurazione adottato da Sørensen e storia della nascita e del significato del simbolo $\mathrm{pH}$}

I primi metodi utilizzati per misurare la $\left[\mathrm{H}^{+}\right]$, o meglio, per misurare l" attività" degli ioni idrogeno, sfruttavano la capacità, posseduta da una soluzione elettrolitica, di generare una forza elettromotrice misurabile mediante una pila galvanica.

Dopo le pionieristiche scoperte sull'elettricità da parte degli italiani Luigi Galvani (1791) e Alessandro Volta (1801), lo svedese Svante Arrhenius enunciò la teoria della dissociazione elettrolitica, nel 1887, e il tedesco Wilhelm Ostwald, nel 1893, rivelò che i fattori decisivi per la generazione della

\begin{tabular}{|c|c|c|}
\hline & {$[\mathrm{H}+] \quad \mathrm{gEq} / \mathrm{L}$} & {$[\mathrm{H}+] \quad \mathrm{gEq} / \mathrm{L}$} \\
\hline$\stackrel{8}{z}$ & 1 & 1.0 \\
\hline 8 & 0,1 & $1.0 \times 10^{-1}$ \\
\hline 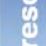 & 0,01 & $1.0 \times 10^{-2}$ \\
\hline Ј & 0,001 & $1.0 \times 10^{-3}$ \\
\hline & 0,0001 & $1.0 \times 10^{-4}$ \\
\hline & 0,00001 & $1.0 \times 10^{-5}$ \\
\hline & 0,000001 & $1.0 \times 10^{-6}$ \\
\hline & neutro 0,0000001 neutro & $1.0 \times 10^{-7}$ \\
\hline$\stackrel{ \pm}{ \pm}$ & 0,00000001 & $1.0 \times 10^{-8}$ \\
\hline छ & 0,000000001 & $1.0 \times 10^{-9}$ \\
\hline & 0,0000000001 & $1.0 \times 10^{-10}$ \\
\hline & 0,00000000001 & $1.0 \times 10^{-11}$ \\
\hline$\frac{\pi}{0}$ & 0,000000000001 & $1.0 \times 10^{-12}$ \\
\hline & 0,0000000000001 & $1.0 \times 10^{-13}$ \\
\hline & 0,00000000000001 & $1.0 \times 10^{-14}$ \\
\hline
\end{tabular}

Figura 5 Due diversi modi di esprimere graficamente gli stessi numeri delle piccole concentrazioni.

corrente elettrica erano costituiti dalla concentrazione degli ioni idrogeno e da quella degli ioni idrossido, nella dissociazione dell'acqua.

È proprio la dissociazione dell'acqua a determinare la quantità di idrogeno libero e l'attività dello stesso. Infatti, in una cella elettrochimica, se si immergono due elettrodi in una soluzione contenente elettroliti gli ioni disciolti migrano verso gli elettrodi a carica opposta e questa migrazione genera una corrente elettrica che può essere misurata.

Il dispositivo sperimentale utilizzato da Sørensen consisteva di due elettrodi, cosi descritti testualmente da lui stesso a p. 20 del suo pionieristico lavoro [3] ( fig. 7): "Per tutte le misure di questo genere, io mi sono servito di uno stesso dispositivo sperimentale. La pila con cui misuravamo la forza elettromotrice consisteva di un elettrodo di calomelano, immerso in una soluzione normale al $10^{\mathrm{e}}$ di cloruro di potassio, e di un elettrodo a idrogeno (placca in platino

\begin{tabular}{|c|c|c|c|}
\hline & pH & {$[\mathrm{H}+] \mathrm{gEq} / \mathrm{L}$} & {$[\mathrm{H}+] \quad \mathrm{gEq} / \mathrm{L}$} \\
\hline & 0 & 1 & 1.0 \\
\hline & 1 & 0,1 & $1.0 \times 10^{-1}$ \\
\hline & 2 & 0,01 & $1.0 \times 10^{-2}$ \\
\hline & 3 & 0,001 & $1.0 \times 10^{-3}$ \\
\hline & 4 & 0,0001 & $1.0 \times 10^{-4}$ \\
\hline & 5 & 0,00001 & $1.0 \times 10^{-5}$ \\
\hline & 6 & 0,000001 & $1.0 \times 10^{-6}$ \\
\hline neutro & 7 & 0,0000001 & $1.0 \times 10^{-7}$ \\
\hline 巴 & 8 & 0,00000001 & $1.0 \times 10^{-8}$ \\
\hline $\bar{\Phi}$ & 9 & 0,000000001 & $1.0 \times 10^{-9}$ \\
\hline 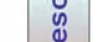 & 10 & 0,0000000001 & $1.0 \times 10^{-10}$ \\
\hline & 11 & 0,00000000001 & $1.0 \times 10^{-11}$ \\
\hline & 12 & 0,000000000001 & $1.0 \times 10^{-12}$ \\
\hline & 13 & 0,0000000000001 & $1.0 \times 10^{-13}$ \\
\hline & 14 & 0,00000000000001 & $1.0 \times 10^{-14}$ \\
\hline
\end{tabular}

Figura 6 Scala del pH e comparazione con i valori numerici delle concentrazioni idrogenioniche, espresse mediante i numeri decimali oppure mediante l'"esponente negativo". 


\section{A. Mesurages électrométriques. \\ a. Méthodes de mesure.}

Pour tous les mesurages de ce genre, je me suis servi d'un même dispositif expérimental. La pile dont on mesurait la force électromotrice, consistait en une électrode au calomel plongée dans une solution norm. au $\mathrm{IO}^{\mathrm{e}}$ de chlorure de potassium et en une électrode à hydrogène (plaque en platine platinée) immergée dans la solution à examiner et qui servait de liqueur électrolyte.

Compt Rend Trav Carlsberg Lab 1909, 8, 1-168 Pag. 20 originale

Figura 7 Testo del lavoro di Sørensen in cui descrive il suo dispositivo sperimentale (fonte: Sørensen SPL. Compt Rend Trav Carlsberg Lab 1909;8:20).

platinata) immerso nella soluzione da esaminare, che serviva da liquido elettrolitico".

Il $\mathrm{pH}$ è misurato utilizzando i due elettrodi, uno come "elettrodo referente" (o "di riferimento") e l'altro come "elettrodo indicatore". Il potenziale dell'elettrodo referente rimane costante ( fixed potential), mentre il potenziale dell'elettrodo indicatore cambia in base ai vari valori di $\mathrm{pH}$ da esaminare; praticamente, il pH-metro misura una forza elettromotoria (o meglio, l'attività elettrica generata) che può anche non corrispondere alla quantità reale dell'acido contenuto nella soluzione, ma è correlata alla sua "attività idrogenionica" (per tale definizione si veda la spiegazione nei paragrafi successivi).

La "soluzione da analizzare" venne indicata arbitrariamente da Sørensen con la lettera alfabetica "p", comparata alla "soluzione di riferimento", indicata, sempre arbitrariamente, con la lettera alfabetica "q" e rappresentata da una soluzione standard a pH noto, reso stabile mediante tamponi.

La "concentrazione di idrogenioni" presenti nella soluzione chiamata " $p$ " venne espressa tipograficamente con il simbolo " $\mathrm{C}_{\mathrm{p}}$ "; essa indicava il numero di grammi-atomi (moli) di ioni idrogeno per litro. Poiché si trattava di numeri molto piccoli, inferiori a 1, e per evitare gli zeri dopo la virgola (molto scomodi nella fase dei calcoli matematici), divenne quasi indispensabile rappresentarli con il numero 10 a esponente numerico negativo $\left(10^{-n}\right)$, con $n$ variabile a seconda della quantità ( fig. 5).

Il passaggio successivo, per evitare lo scomodo esponente negativo, fu quello di esprimerli in chiave logaritmica, cioè indicando con quale numero (da 1 a 14) il fattore base 10 dovesse essere moltiplicato per eguagliare la concentrazione degli idrogenioni (cioè quale dovesse essere l' "esponente" o la "potenza" a cui elevare il numero 10) (fig. 6).

Il simbolo finale utilizzato per indicare tale numero divenne quello rappresentato nella fig. 8 , in cui " $p$ " era il numero in questione, da usare quale esponente negativo per il numero 10 (ovvero, il cosiddetto logaritmo negativo in base 10 della concentrazione degli idrogenioni, cioè la classica definizione del $\mathrm{pH}$ ).

Nella figura è riprodotto l'originale e autentico simbolo del $\mathrm{pH}$, come era stato scritto da Sørensen nel suo lavoro del 1909 (per la prima volta a p. 4 della sua introduzione e poi nelle pagine successive), ovvero con una " $p$ " in carattere minuscolo, affiancata da una lettera " $\mathrm{H}$ " in maiuscolo ma in

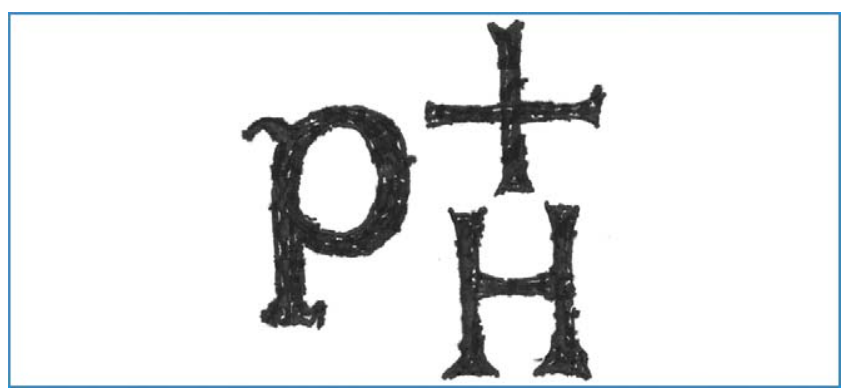

Figura 8 Riproduzione del simbolo originale del pH secondo Sørensen (fonte: Sørensen SPL. Compt Rend Trav Carlsberg Lab $1909 ; 8: 4)$.

posizione cosiddetta di "pedice" (ai piedi della lettera precedente), come comunemente è indicato nel linguaggio della stampa tipografica.

Non si trattava, inoltre, di una " $\mathrm{H}$ " normale isolata: era infatti anche sormontata (non affiancata) dal simbolo " ", a indicare la carica positiva dello ione idrogeno.

Il simbolo $\mathrm{p}_{H}^{+}$, seguito dal numero corrispondente, esprimeva graficamente per Sørensen la quantità (o l'attività) di ioni idrogeno $\left(\mathrm{H}^{+}\right)$presenti nella soluzione da analizzare, indicata, occasionalmente, con la lettera "p".

Sørensen avrebbe potuto chiamare tale soluzione anche "a" o "b" e oggi, al posto del "pH", avremmo parlato di "aH" oppure di "bH".

Questa autentica interpretazione "dell'origine e del significato della piccola $\mathrm{p}$ in $\mathrm{pH}$ " è stata fornita elegantemente da Nørby in un documentato articolo [7] e alla stessa conclusione si può pervenire facilmente rileggendo il lavoro originale di Sørensen.

D'altronde, la scelta della "p" era, all'epoca, una convenzione matematica abbastanza diffusa e l'utilizzo delle due lettere "p" e "q" era molto comune tra i chimici, $i$ matematici, i genetisti e gli altri sperimentatori [8]. Lo stesso Cartesio (1596-1650) aveva già utilizzato le lettere "p" e "q" nel marcare alcuni punti sulle curve da lui disegnate [9] e lo stesso può valere per le lettere dell'elettrocardiogramma $(\mathrm{P}, \mathrm{Q}, \mathrm{R}, \mathrm{S})$ che stranamente furono utilizzate da Einthoven nel 1895 (perché non $A, B, C$ e $D$, per esempio?) [10,11] oppure, ancora, per le branche $\mathrm{P}, \mathrm{Q}$ e R dello spettroscopio o per il galvanometro di Du Bois-Reymond [8].

Questa, quindi, è la vera origine della lettera " $p$ " del $\mathrm{pH}$, anche se oggi, nell'accezione comunemente utilizzata, essa viene interpretata come abbreviazione della parola "potenza" o "potere" nelle varie lingue: puissance in francese, potenz in tedesco, power o potential in inglese, potentia o potens o pondus in latino.

Ovviamente la parola "potenza" non deve essere intesa nell'accezione di "forza" o "potere", ma nel significato matematico di "potenza a cui elevare il numero 10" per eguagliare il valore della concentrazione idrogenionica, cioè nel significato di "esponente" o di "potenziale". Per esempio, 10 elevato alla potenza di 2 , cioè $10^{2}$, oppure alla potenza di 5 , cioè $10^{5}$, oppure, in caso di numeri inferiori a 1, come nel nostro caso, 10 elevato a una potenza con segno negativo (-), dal che la dizione "esponente negativo" 0 ancora "logaritmo negativo in base 10" della concentrazione idrogenionica. 
Lo stesso sito web di Carlsberg Group fornisce la "sua" versione del vero significato, sostenendo che il simbolo "pH" sia l'abbreviazione di potential of Hydrogen e consenta "una semplice e universale misura della quantità di idrogenioni in una soluzione, il che esprime la sua acidità e come gli idrogenioni reagiscano chimicamente in essa".

Dallo stesso sito si evince che il valore del $\mathrm{pH}$ viene misurato, comunemente, durante le fasi operative per la produzione della birra (in cui il controllo dell'acidità è cruciale); il processo di fermentazione viene così monitorato per assicurarsi, in definitiva, che la produzione della birra stia avvenendo secondo le regole [11].

Ovviamente lo stesso vale per tutte le altre molteplici necessità di utilizzo del pH in biologia, in chimica ecc., in quanto è importante anche per determinare la qualità delle acque potabili, per molti prodotti alimentari e per un gran numero di lavorazioni industriali (produzione di carta, di farmaci, tintura, stampa ecc.) o di processi tecnologici (produzione di vino, birra, conservazione di latte, carne ecc.). Nel corso degli anni il simbolo proposto da Sørensen è stato modificato in vario modo, fino a raggiungere la grafica attuale ( $\mathrm{p}$ minuscola e $\mathrm{H}$ maiuscola), cioè $\mathrm{pH}$, da quando fu scelto in maniera definitiva, nel 1917, dalla rivista Journal of Biological Chemistry.

Da allora la grafia non è stata più modificata: potenza degli editori o dei tipografi? Fu una scelta dell'editore o quella di un tipografo, sicuramente stanco di districarsi tra minuscole, maiuscole, pedice e carica ionica soprascritta?

In ogni caso, poiché non potremo mai sapere con estrema precisione che cosa avesse pensato realmente Sørensen e per non fornire personali - e forse false interpretazioni aleatorie del suo pensiero, conviene attenersi alle parole testuali utilizzate dallo stesso inventore nel suo lavoro originale del 1909, ove compare il nuovo simbolo nell'introduzione a p. 4 (fig. 9) e dove, però, Sørensen non rivela le considerazioni che lo indussero a scegliere la lettera "p".

"La grandezza della concentrazione degli ioni idrogeno si esprime mediante il fattore di normalità della soluzione in rapporto agli ioni idrogeno, fattore indicato sotto la forma di una potenza negativa di 10. Per i dettagli, rinvio i miei lettori a un altro capitolo (vedi p. 28); io mi limito a menzionare qui che il valore numerico dell'esponente sarà designato con $\mathrm{p}_{\mathrm{H}}^{+}$e appellato esponente degli ioni idrogeno."

Seguendo l'indicazione di Sørensen e andando a p. $28 \mathrm{del}$ suo scritto, ritroviamo specificato meglio il suo pensiero ( fig. 10): "In tutti i casi trattati nella presente memoria, la $\mathrm{C}_{\mathrm{p}}$ - che significa, in altri termini, il numero di grammiatomi (moli) di ioni idrogeno per litro - è più piccola di $1 \mathrm{e}$ può essere posta uguale a $10^{-\mathrm{p}}$, dove per il numero $p$ io propongo il nome di esponente degli ioni idrogeno e la designazione $\mathrm{p}_{\mathrm{H}}^{+}$. E prosegue: "Per esponente degli ioni idrogeno $\mathrm{p}_{\mathrm{H}}^{+}$di una soluzione, noi intendiamo il logaritmo Briggs del valore reciproco del fattore di normalità della soluzione relativamente agli ioni idrogeno" (concentrazione degli ioni idrogeno con il segno invertito). [In pratica, sintetizzando per $i$ neofiti non chimici, la sigla $C_{p}$ rappresenta la concentrazione degli ioni idrogeno e il logaritmo Briggs è il logaritmo negativo in base 10 di questa concentrazione (logaritmo decimale o volgare o di Henry Briggs). $\mathrm{NdA}]$
Par conséquent, la grandeur de la concentration des ions hydrogène s'exprime par le facteur de normalité de la solution par rapport aux ions hydrogène, facteur indiqué sous la forme d'une puissance négative de I0. Pour les détails, je renvoie mes lecteurs à un autre chapitre (v. p. 28); je me bornerai à mentionner ici que la valeur numérique de l'exposant sera désignée par $p_{H}^{+}$dt appelée exposant des ions hydrogène.

Figura 9 Testo del lavoro di Sørensen in cui, per la prima volta nella storia della medicina, compare il nuovo simbolo del pH nella versione originale (fonte: Sørensen SPL. Compt Rend Trav Carlsberg Lab 1909;8:4).

Dans tous les cas traités dans le présent mémoire, $\mathrm{C}_{\rho}-$ qui signifie, comme nous l'avons dit, le facteur de normalité de la solution sous le rapport des ions hydrogène ou, en d'autres termes, le nombre d'atomes-grammes d'ions hydrogène par litre - est plus petit que $I$ et peut être posé égal à $\mathrm{IO}^{-\mathrm{p}}$, où pour le nombre $p$ je propose le noph 'exposant des ions hy. drogène et la désignation $\left(\mathrm{P}_{\mathrm{H}}^{+}\right.$. Par exposant des ions hydrogène $\left(\mathrm{p}_{\mathrm{H}}^{+}\right)$d'une solution, nels entendons donc le logarithme Brigg de la valeur réciproque du facteur de normalité de la solution relativement aux ions hydrogène $e^{1}$ ).

Figura 10 Testo del lavoro di Sørensen in cui si specifica il significato della concentrazione idrogenionica della soluzione "p" $\left(C_{p}\right)$, nonché il nome da attribuire al simbolo "p" del pH e la spiegazione del logaritmo da esso estrapolato (fonte: Sørensen SPL. Compt Rend Trav Carlsberg Lab 1909;8:28).

Se andiamo, poi, a p. 8 del lavoro di Sørensen, possiamo trovare una delle spiegazioni per cui egli preferì utilizzare $i$ logaritmi ( fig. 11): "In breve, per tutte le reazioni enzimatiche esaminate fino a oggi, si è potuto riconoscere che le modifiche della velocità con cui la reazione avviene dipendono non dalla grandezza assoluta, ma dalla grandezza relativa della modifica della concentrazione ionica. Ne consegue che, negli studi di questo genere, non conviene calcolare con i valori assoluti della concentrazione degli ioni idrogeno, ma con i loro logaritmi, cioè con i suddetti esponenti degli ioni. Infatti, espressa in questo modo, una modifica della concentrazione ionica da $10^{-5}$ a $10^{-4}$ significherà un cambiamento uguale a quello prodotto con una modifica da $10^{-2}$ a $10^{-1}$. In questo modo è possibile rappresentare graficamente l'influenza della concentrazione degli ioni idrogeno, cioè, come abbiamo sopra indicato, per mezzo di curve della concentrazione degli ioni idrogeno. Ciò non sarebbe possibile se l'unità di misura utilizzata in ascissa fosse una quantità qualunque di ioni idrogeno, per esempio quella corrispondente a una concentrazione ionica di $10^{-7}$. In questo caso, le soluzioni aventi concentrazione $10^{-6}$ e $10^{-5}$ da un lato, e quelle $10^{-8}$ e $10^{-9}$ dall'altro, sarebbero rappresentate dalle ascisse 10 e 100 per le prime due e 0,1 e 0,01 per le ultime due, e se la rappresentazione grafica dovesse abbracciare una quantità ancora più grande di concentrazione ionica, le difficoltà sarebbero anche insormontabili”. 


\begin{abstract}
Bref, pour toutes les réactions enzyrnatiques examinées jus qu'à ce jour, on a pu reconnaitre que les modifications de la vitesse avec laquelle la réaction marche, dépendent, non pas de la grandeur absolue, mais de la grandeur relative de la modification de la concentration ionique. Il s'ensuit que dans les études de ce genre, il convient de ne pas calculer avec les valeurs absolues des concentrations des ions hydrogène, mais avec leurs logarithmes, c'est-à-dire, avec lesdits exposants des ions. Exprimée de cette façon, en effet, une modification de la concentration ionique de $\mathrm{IO}^{-5}$ à $\mathrm{IO}^{-4}$ signifiera un changement égal à celui produit par une modification de $10^{-2}$ à $10^{-x}$. Aussi, par cette voie est-il possible de représenter graphiquement l'influence de la concentration des ions hydrogène, c'est-à-dire, comme nous l'avons indiqué plus haut, au moyen des courbes de la concentration des ions hydrogène. Ceci ne serait guère possible, si l'unité des abscisses était une quantité absolue quelconque d'ions hydrogène, par ex., celle correspondant à une concentration ionique de $\mathrm{IO}^{-7}$. En ce cas, des solutions ayant les concentrations $1^{-6}$ et $\mathrm{IO}^{-5}$, d'un côté, et, de l'autre, $\mathrm{IO}^{-8}$ et $\mathrm{IO}^{-9}$, seraient représentées par les abscisses Io et IoO pour les deux premières solutions et $\mathrm{O}, \mathrm{I}$ et $\mathrm{O}, \mathrm{O}$ I pour les deux dernières, et si la représentation graphique devait embrasser une étendue encore plus grande de concentration ionique, les difficultés seraient même insurmontables.
\end{abstract}

Figura 11 Testo del lavoro di Sørensen in cui l'autore spiega le ragioni della scelta dei logaritmi (fonte: Sørensen SPL. Compt Rend Trav Carlsberg Lab 1909;8:8).

\section{In medicina è proprio indispensabile utilizzare i logaritmi negativi per misurare gli idrogenioni?}

Il logaritmo (per giunta, anche negativo) era l'unica possibilità per eliminare i numeri inferiori a 1 con tanti zeri dopo la virgola? La risposta è sicuramente: no! E non è difficile dimostrarlo.

Se la fantasia dei ricercatori fosse stata più "banale" e meno "ricercata", la soluzione del problema si sarebbe potuta trovare nell'utilizzare, al posto del $\mathrm{pH}$, una nuova unità di misura che sostituisse $\mathrm{i}$ grammi-atomi per litro $\mathrm{e}$ anche i milliequivalenti per litro $(\mathrm{mEq} / \mathrm{L})$.

L'uovo di Colombo sarebbe stata (come poi è avvenuto) l'invenzione del micromilliequivalente, più simpaticamente appellato nanoequivalente $(\mathrm{nEq})$ che all'epoca, forse, non esisteva ancora ( fig. 12). Con questa nuova unità di misura il valore della neutralità corrisponde a $100 \mathrm{nEq}$; il valore normale della concentrazione idrogenionica del sangue umano è 40.

Infatti, se viene espresso in grammi, il valore normale $(7,40)$ del $\mathrm{pH}$ è pari a $0,00000004 \mathrm{~g} / \mathrm{L}$, con sette zeri dopo la virgola seguiti dal numero 4 (il che corrisponde a un numero esponenziale negativo di $1,0 \times 10^{-7,4} \mathrm{e}$, cioè, a un pH di 7,40).

Considerato che il "grammo" è un miliardo di volte più grande del "nanogrammo" ( fig. 13), se si trasforma questo numero in nanoequivalenti si ottiene il semplice numero 40 (spostando la virgola di nove volte verso destra), il che è molto più facile da gestire rispetto sia al lungo numero decimale e al $\mathrm{pH} 7,40$, sia al numero esponenziale negativo $1,0 \times 10^{-7,4}$.

Operando le dovute sostituzioni, come nella fig. 14 , si ha un tipo diverso di scala della concentrazione idrogenionica, con numeri più "umani", di tipo matematico semplice, più popolari, più alla portata di uno studente di media cultura e

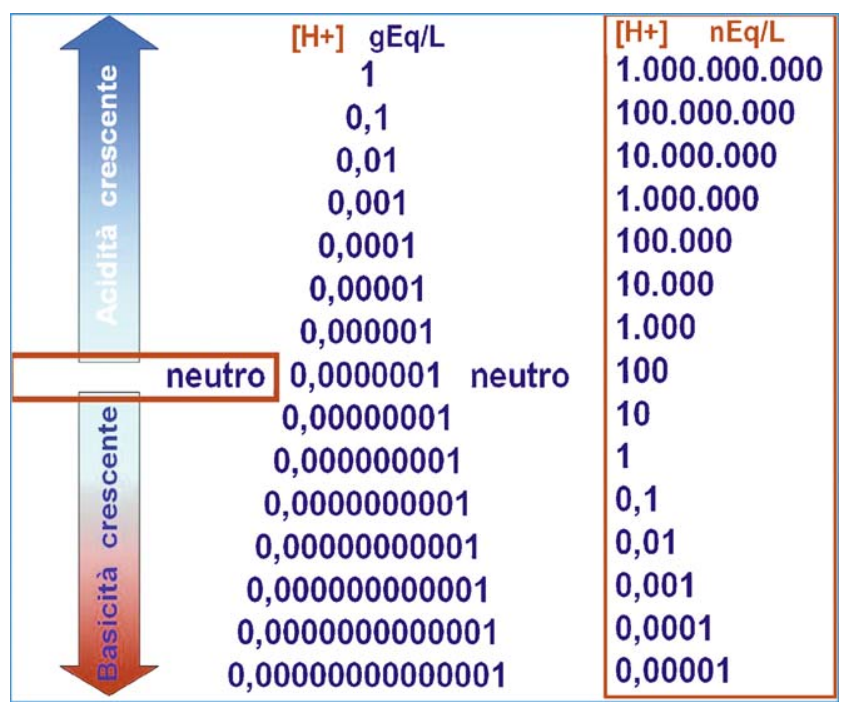

Figura 12 Comparazione tra la scala espressa in grammiequivalenti e quella espressa in nanoequivalenti.

di medio amore verso la matematica e la chimica, senza le complicazioni logaritmiche, indiscutibilmente antipatiche, e soprattutto senza l'esponente negativo.

Grazie all'utilizzo dei nanoequivalenti, tutti i ragionamenti vengono portati avanti in chiave lineare, con un rapporto diretto (non invertito) tra l'aumento degli idrogenioni e il corrispondente aumento dei nanoequivalenti, a espressione dell'aumento dell'acidità della soluzione in studio (sangue o altro). Con questa scala, infatti, più aumentano gli idrogenioni più aumentano i nanoequivalenti (e viceversa nel senso della diminuzione).

È indiscutibile che il numero 40 sia molto più maneggevole (eliminate le inutili sovrastrutture mentali) e, nel caso specifico del sangue umano, le evidenze a favore di questa tesi sono ancora più incontrovertibili, perché il range della compatibilità con la vita, e quindi il range dello studio, è ancora più ristretto. È ben noto, infatti, che l'ambito di sopravvivenza umana compatibile con una particolare concentrazione idrogenionica oscilla tra i valori di $\mathrm{pH} 7,8$ e 6,8. Al di fuori di tale range i metabolismi cellulari si bloccano, le reazioni enzimatiche ribosomiali cessano e la vita dell'organismo si interrompe ( fig. 15).

Nella fig. 15 si evidenzia chiaramente che la condizione di neutralità ionica $(\mathrm{pH} \mathrm{7,0)}$ coincide con il valore di $100 \mathrm{nEq}$, mentre il valore normale fisiologico del sangue umano $(\mathrm{pH}$ 7,40 ) corrisponde a $40 \mathrm{nEq}$ e il valore di $\mathrm{pH} 8$ corrisponde a 10

\begin{tabular}{|l||ll|}
\hline $\mathrm{gEq}$ & 1 & unità \\
$\mathrm{mEq}$ & $1 / 1.000$ & millesimo \\
$\mu \mathrm{Eq}$ & $1 / 1.000 .000$ & milionesimo \\
$\mathrm{nEq}$ & $1 / 1.000 .000 .000$ & miliardesimo \\
$\mathrm{pEq}$ & $1 / 1.000 .000 .000 .000$ & bilionesimo \\
\hline
\end{tabular}

Figura 13 Comparazione delle unità di misura: il nanoequivalente $(\mathrm{nEq})$ è la miliardesima parte del grammo. 


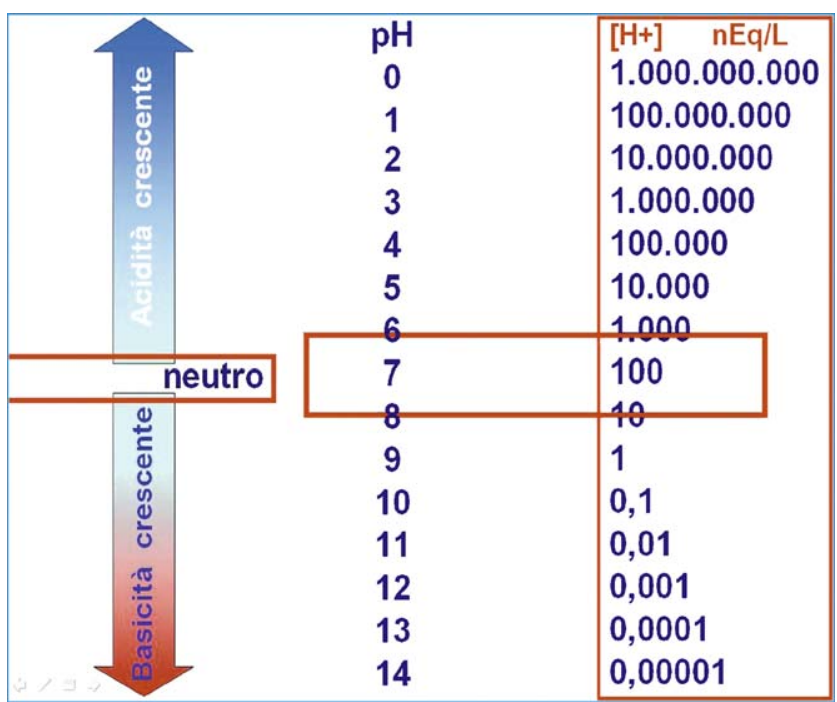

Figura 14 Comparazione fra il pH e la concentrazione idrogenionica espressa in nanoequivalenti nello stretto range della possibile sopravvivenza umana.

nEq. Gli estremi compatibili con la vita (pH 7,80 e pH 6,80) sono espressi, in termini di nanoequivalenti, dai numeri $16 \mathrm{e}$ 160 , molto familiari a tutti.

In questa maniera, anche i meno aggiornati in tema di cultura generale e con scarsa attrazione verso la matematica sono in grado di capire con facilità che più aumentano $i$ nanoequivalenti più aumenta l'acidità, con un rapporto numerico lineare che può essere seguito anche nelle variazioni di singole unità rispetto alla norma (come per esempio $40,41,42$ ecc.), e ovviamente, nel senso inverso della scala, più diminuiscono i nanoequivalenti $(39,38,37$ ecc.) più diminuisce l'acidità.

Questo modo di esprimere la concentrazione idrogenionica consente a chiunque di valutare facilmente l'entità dell'eventuale anomalia rispetto alle condizioni fisiologiche, come avviene per la glicemia, la sodiemia, la potassiemia ecc., grazie all'utilizzo della semplice unità di misura decimale.

D'altronde, gli apparecchi per la valutazione del $\mathrm{pH}$ (pH-metri) misurano la "concentrazione" (o l' "attività" degli idrogenioni, si veda il paragrafo successivo), e solo successivamente questi numeri vengono trasformati (dal computer installato all'interno della macchina) in chiave logaritmica, il che oscura la grandezza dei cambiamenti e può indurre a sottovalutare l'entità delle variazioni. Basterebbe, invece, che l'apparecchio analizzatore ci fornisse il numero originario degli idrogenioni espressi semplicemente sotto forma di nanoequivalenti.

Nell'attuale situazione ("logaritmica") del pH, però, l'apprendimento che poteva essere semplice è diventato materia di élite. Tuttavia la medicina non può e non deve essere elitaria, perché "gioca" con la vita delle persone e tutti, quindi, devono essere in grado di capire bene e in maniera rapida il quadro clinico che hanno davanti, senza complicazioni superflue e inutili, evitabili facilmente senza compromettere il rigore scientifico. Tra l'altro, l'impostazione logaritmica risulta misteriosa e incomprensibile anche per gli stessi "iniziati", i cosiddetti esperti, considerato che la letteratura è ricca di commenti ironici o drastici nei

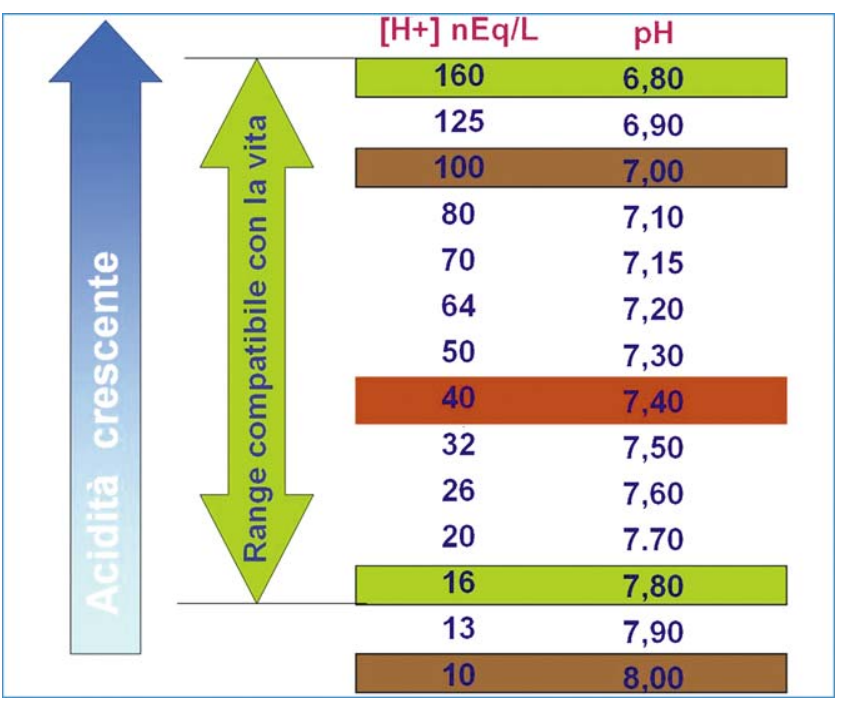

Figura 15 Lo stretto range di compatibilità con la vita.

confronti di questo tipo di didattica (e la nostra condivisione è totale).

"Con tutto il rispetto per il brillante contributo di S.P.L. Sørensen alle nostre conoscenze sul pH e sui tamponi, l'insegnamento continuo del concetto di $\mathrm{pH}$ è stato un disastro educazionale" [12].

"Questo metodo didattico di insegnamento dell'equilibrio acido-base, secondo l'utilizzo dell'equazione di HendersonHasselbalch con i logaritmi, ha mostrato indiscutibilmente un evidente fallimento" [13].

"L'oggetto di questa relazione costituisce uno degli argomenti più ostici della scienza medica" [14].

"Il concetto di pH è una delle idee più confuse in chimica e in biochimica" [15].

"Alcuni lo trovano un rompicapo e, senza dubbio, la loro nebbia ha qualcosa a che fare con quel logaritmo negativo" ("Some find it a puzzlement. Doubtless their fog/Has something to do with that negative log") [16]. In questo caso l'autrice, di lingua inglese, ha trovato finanche un'assonanza in rima fra le parole "nebbia" ( $f \circ g)$ e "logaritmo" (log).

"Quando Dio creò il mondo, cominciò, presumibilmente, con l'idrogeno. L'idrogeno è il primo elemento della tavola periodica [...] In principio c'era il caos e l'idrogeno, alla fine rimasero, tra gli altri risultati della creazione, l'equilibrio acido-base e il medico spesso confuso" [17].

"Qui si gioca la vita dell'uomo, eppure i medici si comportano verso l'equilibrio acido-base come molti cattolici verso la Bibbia: ne hanno un grande rispetto, e questo rispetto lo dimostrano standone il più lontano possibile" (Paul Claudel, scrittore francese, citato da Gianfranco Ravasi, grande studioso di testi sacri) $[18,19]$.

"L'equazione di Henderson è ovviamente più facile da usare rispetto alla classica formula di HendersonHasselbalch, per il fatto che non contiene logaritmi" [20].

"Quando parliamo di incrementi o di decrementi dell'acidità, il $\mathrm{pH}$ varia inversamente alla $[\mathrm{H}+]$. Questo è confondente" [21].

"Molti studenti vengono confusi, principalmente, dal fatto che i più alti valori di $\mathrm{pH}$ corrispondono alle più basse acidità. Questo è solo un problema minore nell'insegnamento 
del pH [...] La concentrazione idrogenionica è, almeno, una quantità reale che gli studenti possono capire" [2].

"Vi sono probabilmente pochi aspetti della medicina che procurano al medico medio maggiori difficoltà dei disturbi dell'equilibrio acido-base. Nello sforzo di semplificare l'analisi di questi disturbi sono stati pubblicati diversi nomogrammi, ma il loro alto numero testimonia la loro apparente mancanza di successo" [20].

"L'origine del simbolo $\mathrm{pH}$ ha confuso generazioni di studenti, fotografi e giardinieri" [22].

"Nell'organismo umano, in fondo, ci sono gli idrogenioni e non i logaritmi degli idrogenioni, che i medici, notoriamente, non sanno calcolare" [23,24].

"Sfortunatamente, il pH sopravvisse e ha continuato a sconcertare generazioni di studenti in medicina. Comunque, nell'era della nanotecnologia, il concetto di una concentrazione nanomolare può ora mostrarsi più accettabile e può aiutare a demistificare il capitolo dell'equilibrio acido-base" [25].

"Se il medico non è naturalmente incline alla chimica [e, ahimè, questo è il caso di molti di noi! NdA], egli si fa venire la pelle d'oca e gira al largo verso un'altra via quando tali fenomeni, come acidi, basi, tamponi, curve di titolazione e $\mathrm{pH}$, si profilano all'orizzonte medico" [17].

"La materia è ostica non tanto per la difficoltà, quanto per il fatto che la maggior parte dei medici ha abbracciato la medicina in odio alla matematica, alla chimica e ai numeri in genere, e prova una ripulsa quando è costretto ad affrontare problemi nei quali compaiono operazioni diverse dalle quattro elementari" [26].

"In nessun capitolo della biologia e della medicina vi è una confusione più sfrenata e una chiarezza concettuale più difficile da ottenere che nel capitolo dell'equilibrio acidobase" [27].

La natura umana si deve difendere dall'eccesso degli idrogenioni, che sono misurabili come tutte le altre sostanze in termini di quantità, oppure anche in termini di attività elettrica, ma devono sempre essere espressi con unità di misura semplici e comprensibili da tutti. Nessuno si sognerebbe, oggi, di "semplificare" la rappresentazione della concentrazione del sodio o del potassio o della glicemia adottando come unità di misura il logaritmo del reciproco dei loro valori. Questo, invece, è quanto viene fatto, da un secolo, per la concentrazione dello ione idrogeno, che è l'unico a essere rappresentato così, tra tutti gli altri componenti del sangue umano analizzati in laboratorio, $i$ quali vengono, invece, misurati in termini di "quantità per unità di volume", con numeri semplici decimali e senza "inversioni reciproche" o altri equilibrismi a testa in giù.

Tra l'altro, poi, nel range della normale vivibilità $(\mathrm{pH}$ compreso tra 6,8 e 7,8) il rapporto fra i nanoequivalenti e i valori del $\mathrm{pH}$ mantiene una correlazione quasi perfettamente lineare ( fig. 16), il che consente facilmente di passare da una scala all'altra; non esiste perciò alcuna necessità di "complicarsi" la vita utilizzando la scala più contorta, cioè quella logaritmica. Questa via disagevole per tutti, quindi, è pressoché "superflua" in medicina e, in aggiunta, finisce per diventare "dannosa" per molti pazienti.

"La precisione con cui i moderni apparecchi sono in grado di misurare $\mathrm{i}$ valori del $\mathrm{pH}$ supera la capacità delle nostre conoscenze per interpretare esattamente ciò che $i$ numeri significano" [16]. Questa frase fu enunciata nel 1979 e oggi,

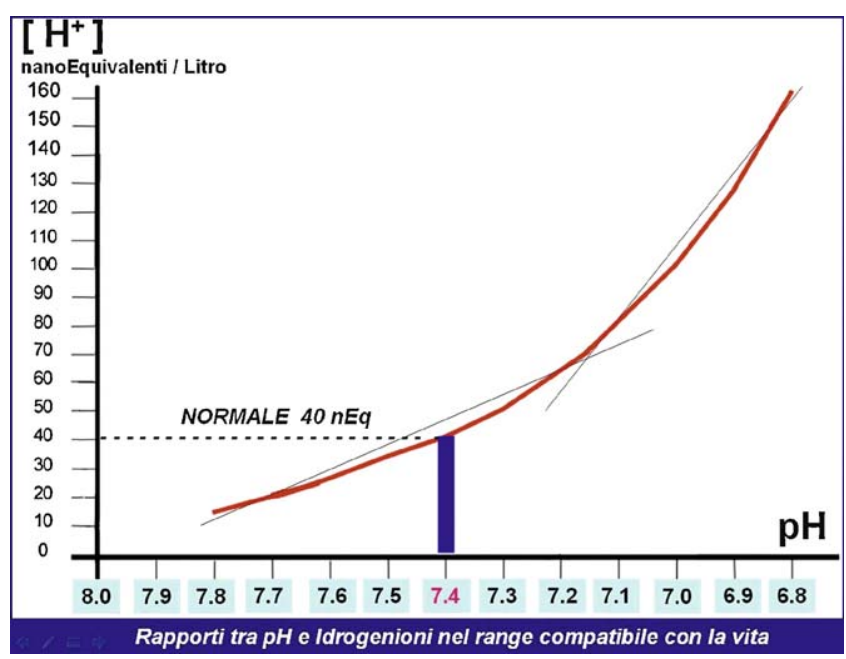

Figura 16 Rappresentazione grafica dei rapporti quasi lineari (non logaritmici) tra il pH e gli idrogenioni, nell'ambito del range compatibile con la vita.

anche se sono migliorate moltissimo le strumentazioni analitiche per misurare gli idrogenioni [28], permangono le stesse problematiche interpretative dei dati messi a nostra disposizione.

In definitiva, alla domanda posta nel titolo, "In medicina è proprio indispensabile utilizzare i logaritmi negativi per misurare gli idrogenioni?", la nostra risposta finale è: "No!".

\section{Conflitto di interesse}

Gli autori dichiarano di essere esenti da conflitto di interessi.

\section{Bibliografia}

[1] Holter H, Moller KM. The Carlsberg Laboratory 1876-1976. Carlsberg Foundation. Copenhagen: Rhodos; 1976.

[2] Myers RJ. One hundred years of $\mathrm{pH}$. Journ Chem Educ 2010;87(1):30-2.

[3] Sørensen SPL. Études enzymatiques. II. Sur la mesure et l'importance de la concentration des ions hydrogène dans les réactions enzymatiques. Compt Rend Trav Carlsberg Lab 1909;8:1-168.

[4] Sørensen SPL. Enzymstudien. II: Mitteilung. Über die Messung und die Bedeutung der Wasserstoffionenkoncentration bei enzymatischen Prozessen. Biochem Zeitschr 1909;21:131-304.

[5] Sørensen SPL. Enzymstudien. II: Mitteilung. Über die Messung und die Bedeutung der Wasserstoffionenkoncentration bei enzymatischen Prozessen. Biochem Zeitschr 1909;22:352-6.

[6] Sørensen SPL. Enzymstudier II. Om Maalingen og Betydningen af Brintionkoncentrationen ved enzymatiske Processer. Meddelelser fra Carlsberg Laboratoriet 1909;8:1-168.

[7] Nørby JG. The origin and the meaning of the little $\mathrm{p}$ in $\mathrm{pH}$. Trends Biochem Sci 2000;25(1):36-7.

[8] Myers RJ. Who Put the "p" in the pH Symbol and Why Was It Chosen? In: Book of Abstracts. Proceedings of the $219^{\text {th }}$ ACS National Meeting. San Francisco, CA: 2000.

[9] Hurst JW. Naming of the waves in the ECG, with a brief account of their genesis. Circulation 1998;98(18):1937-42.

[10] Why is that ECG has PQRST waves and not other alphabets? http://answers.yahoo.com/question 
[11] Carlsberg Group. Sørensen Invents the pH Scale. http://www. carlsberggroup.com/Company/Research/Pages/pHValue.aspx

[12] Seeman P. The pH concept. Science 1972;177(4052):835-6.

[13] Po HN, Senozan NM. The Henderson-Hasselbalch equation: Its history and limitations. J Chem Educ 2001;78(11):1499-503.

[14] Polli E, Fieschi A, Bianchi P. Le alterazioni dell'equilibrio acidobase. In: Atti del $74^{\circ}$ Congresso Società Italiana Medicina Interna. Roma: Pozzi; 1973.

[15] Ferguson JB. Exercise 4: Titration of an Amino Acid. http:// biology.bard.edu/ferguson/course

[16] Kolb D. The pH concept. J Chem Education 1979;56:49-53.

[17] Elkinton JR. Hydrogen-ionized and otherwise. Ann Int Med 1962;57(4):687-8.

[18] Sgambato F. Un nuovo teorema. Ovverosia può la filosofia napoletana essere applicata all'equilibrio acido-base? Ah saperlo, saperlo! Eur Resp News 1995;3(2):45-8.

[19] Sgambato F. L'equilibrio acido-base nella pratica clinica senza logaritmi. Relazione al III Congresso Nazionale FADOI, maggio 1998. Internista Ospedaliero 1999;3-4:21-7.

[20] Atkins EL. Assessment of acid-base disorders. A practical approach and review. Can Med Assoc J 1969;100(21):992-8.
[21] Clarke WM. The Determination of Hydrogen lons, $3^{\text {rd }}$ Ed., Baltimore, MD: Williams \& Wilkins; 1928. p. 36-38.

[22] Saxby G. Photography encyclopedia: pH. In: Lenman R, editor. The Oxford Companion to the Photograph. Oxford: Oxford University Press; 2005.

[23] Sgambato F, Prozzo S, Caporaso C. L'equilibrio acido-base nella pratica clinica senza logaritmi. Atti del V Seminario "Equilibrio acido-base e idroelettrolitico". Incontri al Fatebenefratelli, Benevento 1998; pp. 51-65.

[24] Sgambato F, Prozzo S, Milano L, Sgambato E, Piscitelli GL, Fucci A. Gli equilibri omeostatici in Medicina: dalla fisiopatologia alla filosofia. Conferenza all'Accademia Lancisiana. Policlinico Sez Prat 2009;116:1146-66.

[25] Gibson GJ. Moran Campbell and clinical science. Thorax 2004;59:737-40.

[26] Bartoli E. Fisiopatologia e clinica degli squilibri idro-elettrolitici. Medicina Internazionale 1976;23:1-108.

[27] Elkinton JR. Hydrogen ion turnover in health and in renal disease. Ann Int Med 1962;57(4):660-85.

[28] Camões MF. The quality of pH measurements 100 years after its definition. Accredit Qual Ass 2009;14(10):521-3. 